\title{
Skin Alterations Caused by Chemotherapies and Cosmetic Strategies for Soothing These Effects: A Review
}

\author{
Anne Lore Moreira ${ }^{1, *}$, Helena Ross Forneck ${ }^{1}$ and Lucas Portilho ${ }^{2}$ \\ ${ }^{1}$ Pharmacist, Biochemical Engineer and MBA Student at Educational Cosmetology Institute, Brazil \\ ${ }^{2}$ Director, Educational Cosmetology Institute, Brazil
}

*Corresponding author: Anne Lore Moreira, Pharmacist and MBA Student at Educational Cosmetology Institute, Brazil, E-mail: anne.lore.moreira@ gmail.com

Received: 19 Jul, 2021 | Accepted: 04 Oct, 2021 | Published: 15 Oct, 2021

Citation: Moreira AL, Forneck HR, Portilho L (2021) Skin Alterations Caused by Chemotherapies and Cosmetic Strategies for Soothing These Effects: A Review. J Clin Cosmet Dermatol 5(2): dx.doi.org/10.16966/2576-2826.166

Copyright: (C) 2021 Moreira AL, et al. This is an open-access article distributed under the terms of the Creative Commons Attribution License, which permits unrestricted use, distribution, and reproduction in any medium, provided the original author and source are credited.

\begin{abstract}
Cancer is a set of more than one hundred diseases caused by the development of malignant tumors, formed by cells that stand out for their uncontrolled division, altered response to growth factors, increased longevity, changes in function and structure, less intracellular adhesion and ability to invade other tissues in the body. One of the most used treatments currently against these diseases is chemotherapy. It attacks cells that have high rates of division and, therefore, its side effects are felt especially in tissues that naturally present this characteristic, such as skin, nails and hair. Since the affected tissues are deeply linked to beauty and self-esteem, it is necessary to seek alternatives to mitigate the negative effects of the treatment to increase the quality of life of the patients subjected to it. Based on this theme, the objective of this study is to review the physiology of the skin, how malignant tumors appear, the cutaneous side effects of chemotherapy treatments, cosmetic actives present in the Brazilian market indicated to alleviate these effects and suggest the formulation of products for palliative treatment of patients undergoing cancer treatment.
\end{abstract}

Keywords: Cosmetics; Chemotherapy; Skin; Palliative treatment; Tissue repair; Topical

\section{Introduction}

Patients undergoing chemotherapy treatment often receive multiple systemic medications. During the chemotherapy period, several adverse skin reactions develop, ranging from mild erythema, severe skin irritation to tissue necrosis $[1,2]$.

Despite the benefits of these chemotherapeutic agents, toxic effects on the skin can result in poor patient compliance or can cause the patient to cease the antineoplastic therapy altogether. These toxic adverse effects on the skin can also significantly reduce the quality of life these patients experience [3-5].

Most adverse reactions are considered dose dependent, causing manifestations such as alopecia, skin hyperpigmentation, phototoxicity, nail dystrophies, hand-foot syndrome, neutrophilic eccrine hidradenitis and extravasation lesions [2,6-10].

Assessing the skin physiology of cancer patients is challenging as they are exposed to a variety of medications and usually have a poor general condition under immunosuppression [10].

It has been proven that the proper use of topical treatments improves the barrier function of the epidermis and can reduce the likelihood of further worsening of symptoms by preventing dry skin and maintaining its smoothness [1].
Thus, the objective of this study is to present cosmetic strategies for patients undergoing chemotherapy treatments in order to reduce the severity of their adverse reactions.

\section{Materials and Methods}

An electronic literature search was performed using the PUBMED and MEDLINE database. In search of data, the used terms were: chemotherapy, skin, cosmetics, cancer, chemotherapy effects on the skin, topical, tissue repair. These terms were searched in English and Portuguese. The inclusion criteria were articles that evaluated skin changes resulting from chemotherapy, cosmetics that presented palliative benefits for sensitized skin after chemotherapy, and bibliographic reviews of the skin structure and cancer formation. In total, 20 articles published in scientific journals were used as reference for the present review. Additionally, 12 online articles from known reputable sources (government institutes, renowned Universities, and the Mayo Clinic) are listed in the references. 7 academic books were also used as a complementary way for the bibliographic review of the skin structure, such books being mentioned in the references. A market research was carried out on the current products for this target audience (patients with skin sensitized by chemotherapy treatments) on Google, always relating to the scientific data of the products offered. The UL Prospector tool and catalogs of suppliers of raw materials were also used to consult their characteristics. 
Since approximately $28.4 \%$ of all cancer patients are subjected to chemotherapy as primary treatment [11], for the purposes of this review no specific nosological form was favored. Adverse effects linked to specific chemotherapeutic drugs and protocols were identified as the focal point of analysis.

The main sources considered regarding adverse skin effects from chemotherapy are listed in the references and were published in Plastic Surgery Nursing Journal Online, Seminars in Oncology Nursing, and Medical Clinics of North America Journals [12-14].

In the present study, the main focus was to review the literature to relatively analyze the use of cosmetics as a palliative means for patients undergoing chemotherapy treatments, resulting in improved quality of life.

\section{Discussion}

\section{Healthy skin}

Skin structure: Skin is the outer lining of the human body [15]. It is divided into epidermis, dermis and subcutaneous tissue, the latter subdivision being considered as a skin attachment by some authors and not as part of the skin structure itself.

Known for being the largest organ in the human body, it has functions such as: protection, thermoregulation, perception of sensations, vitamin D production, conservation of homeostasis, healing, lipid reserve and secretion of metabolic residues [15]. Its appearance influences self-esteem, social relationships and quality of life, reflecting health and beauty [16].

Of ectodermal origin [15], the epidermis is always in continuous renovation, being composed of the corneum (horny), lucid, granular, spinosum and basale layers. Its cellular constitution is mainly made up of keratinocytes, whose function is the production of keratin - a protein that helps in the protective properties of the skin. These cells originate from the deepest layer of the epidermis, the basale stratum, undergo mitosis continuously and thus differentiate progressively until reaching the stratum corneum, where they become highly keratinized [17].

The desquamation process takes place daily, and the complete renewal of the epidermis occurs in approximately 40 days [18].

The main events during this renewal process are: increase in cell size and flattening, appearance of new organelles and reorganization of existing ones, changes in cell metabolism related to the keratinization process, changes in the properties of the cell membrane, and dehydration $[18,19]$.

The last layer of differentiation of keratinocytes, the horny layer, is the most superficial of the epidermis. Keratinocytes now have the appearance of overlapping slides and are eliminated from time to time. The main function of this layer is to protect the remaining living layers of the skin from the entry of external aggressors, and the loss of transepidermal water. The rupture of these skin barrier functions can trigger the compromise of the structure, causing injuries [15].

Natural repair mechanisms: the skin healing process: The skin healing process is a natural reaction to tissue damage and consists of a cellular events cascade that generates skin remodeling, repair and regeneration. There are four possible types of response to an injury:

a) Regeneration, which is the exact replacement of the injured tissue;

b) Normal repair that reconstitutes the balance between scar tissue formation and tissue remodeling; c) Poor healing that causes the formation of chronic ulcers;

d) Excess healing that causes fibrosis and contractures.

This process starts immediately after the injury has occurred, when there is still bleeding and the platelets come into contact with collagen and other elements of the Extracellular Matrix (ECM) [20].

Skin repair has three phases: inflammation, cell proliferation and deposition of ECM (Extracellular Matrix), and remodeling. In the inflammatory phase, rupture, erosion or fissure of the endothelium directs a sequence of events, where platelet deposition begins, proceeds with its activation and subsequent recruitment of new platelets. The formation of a thrombus with platelets is the result of this sequence, which temporarily buffers the endothelial lesion [21], which is quickly infiltrated by fibrin, becoming a fibrinous thrombus. Then, this fibrous thrombus will capture erythrocytes and form the red thrombus, which is mainly responsible for the occlusion of the ruptured blood vessel [22].

The second phase, cell proliferation, covers the formation of capillaries and ECM to fill the defect caused by the debridement of the lesion, that is, the removal of devitalized tissues to prepare the wound support for its definitive coverage. Reepithelialization is marked by the convergence of keratinocytes at the edges of the lesion and at the basal layer, coming from the differentiation of stem cells from the hair follicles and apocrine glands [23].

To cover the lesion, keratinocytes will migrate to it and proliferate, forming a dense epithelial layer. Other keratinocytes will also migrate and be deposited successively on this cell layer and then fill the injury [23]. The release of growth factors at the site by fibroblasts, macrophages and neutrophils is important for the activation of keratinocytes in the damaged area [24].

With the advancement of the cell proliferation phase, the type of cell that will become permanent is the fibroblast, responsible for the production of the new matrix necessary for the structural and functional restoration of the injured tissue. These cells bind to the provisional fibrin matrix and begin to produce collagen, type I being predominant [20].

Finally, the last phase of the healing process is remodeling, which consists of the gradual degeneration of granular tissue and the regeneration of the dermis. In this phase, the apoptosis of myofibroblasts, endothelial cells and macrophages occurs [25].

\section{Neoplasms in general}

Definitions and origin: Cancers, malignant tumors or neoplasms can appear in different parts of the body and their behavior will be different depending on the organ of origin, but their characteristics do not change when there is metastasis [26-28].

Malignant cell studies show that tumor initiation is linked to genetic mutations at key points in the DNA and the exacerbated proliferation of this single mutant cell $[29,30]$.

There are three types of DNA mutations: base replacement, deletion and insertion. Furthermore, mutations by deletion and insertion can also occur on a single strand, with an infinite range of results [31].

DNA repair mechanisms: a) Direct reversal of DNA damage: In humans, this type of repair is carried out by enzymes capable of transferring the affected groups to their active sites, removing the alteration that has mutagenic potential $[30,32]$.

b) Excision repair mechanism: In this mechanism, the nondefective complementary strip is used as a model, the altered bases 
are removed and replaced by new nucleotides through the action of specialized enzymes [30-33].

c) Post-replication repair: The synthesis of Okazaki fragments below the mutation region allows replication to continue, resulting in a daughter strand with a fragment in lieu of the defect in the mother stripe is found. These fragments are then filled [30,32].

Clinical manifestations: Exposure to promoters - carcinogens capable of stimulating cell proliferation - can be the difference between the growth and the disappearance of a tumor. Important examples of tumor promoters include hormones, radiation and some types of viruses $[30,34]$.

Once established, a malignant tumor will grow and spread until it is noticed. The most common symptoms include fever, fatigue and unexplained weight loss, as malignant cells require a large sum of the energy available in the body [35].

Treatment: Currently, the most used techniques are chemotherapy, radiotherapy and surgery. Other treatments include immunotherapy, targeted therapy, hormone therapy, stem cell transplants and precision medicine [36,37].

When a treatment aims to completely remove the malignant tumor and cancer cells, it is called a primary or definitive treatment. Adjuvant and neoadjuvant treatments, in turn, are those with the objective of removing malignant cells after or before a primary treatment, respectively. Finally, palliative care is aimed at mitigating the symptoms of diseases and/or side effects of other therapies [37].

Side effects of chemotherapy on the skin: Because they target cells with accelerated division, chemotherapy drugs will affect any other type of cell that is undergoing rapid division. As a result, skin reactions are routinely developed during this type of treatment, as cancer cells and skin are subjected to accelerated processes of cell division [12].

These skin reactions range from mild erythema to severe skin irritation and even epidermal necrosis. Most of these reactions are considered dose dependent. Patients often experience adverse skin effects such as xerosis, tenderness and dry skin [12].

According to the consulted bibliography, the adverse effects can be divided into: [12-14,38]

Cytotoxic reactions: stomatitis, alopecia, Beau's lines and skin lacerations; Onycholysis is most commonly seen in patients treated with paclitaxel and docetaxel but also with cyclophosphamide, doxorubicin, 5-FU, hydroxyurea and the combination of bleomycin plus vinblastine. Drugs most commonly correlated with alopecia are bleomycin, daunomycin, cyclophosphamide, dactinomycin, doxorubicin, 5-fluorouracil, hydroxyurea, mitomycin, mechlorethamine, methotrexate, vinblastine, and vincristine. Mucositis could appear in relation to many chemotherapeutic agents but commonly occurs with the administration of bleomycin, cytarabine, dactinomycin, daunorubicin, doxorubicin, 5-fluorouracil, methotrexate. Mucositis can result in stomatitis.

Local injuries: Phlebitis and chemical inflammation under the skin; Phlebitis may occur with the administration of carmustine (BCNU), daunomycin, doxorubicin, 5-fluorouracil and mechlorethamine. Methotrexate can result in the formation of antigen-antibody complexes which cause vasculitis (inflammation surrounding blood vessels).

Hypersensitivity reactions: Urticaria, angioedema, erythema multiforme, toxic necrolysis of the epidermis; L-Asparaginase is the drug that most commonly induces immediate hypersensitivity.
Cisplatin and carboplatin can cause IgE-mediated hypersensitivity with itching, redness and swelling occurring within an hour after the infusion is begun.

Interactions with radiation: Radiation Recall, photosensitivity, reactivation of erythema induced by UV light; Photosensitivity is related with several agents as 5-fluorouracil, dactinomycin, doxorubicin, bleomycin, dacarbazine, hydroxyurea, and vinblastine. Patients receiving high-dose methotrexate could display severe sun reactions after exposure to ultraviolet radiation and are highly encouraged to avoid sun exposure after treatment. Methotrexate can cause a photo enhancement in which administering the drug several days after sunburn causes the sunburn to reappear.

Changes in pigmentation: Hyperpigmentation in the gums, nail, skin, veins; Cytotoxic drugs such as alkylating agents or tumordirected antibiotics cause pigmentary changes. Hyperpigmentation of the gums can be found with cyclophosphamide treatment and is permanent. 5-Fluorouracil (5-FU) treatment is frequently seen with hyperpigmentation reactions in all areas of skin or in only sun-exposed areas. Other drugs may result in generalized hyperpigmentation. Daunorubicin can cause hyperpigmentation in solar-exposed areas. Cisplatin, hydroxyurea and bleomycin cause increased pigmentation in areas of injury or pressure. Docetaxel, thiotepa and ifosfamide are secreted in sweat and can cause hyperpigmentation under adhesive tape. Scalp hyperpigmentation can be associated with Daunorubicin.

Other various reactions: Dactinomycin "acne", Bleomycin nodules (localized nodules and infiltrated plaques appearing on the extremities and buttocks with the administration of Bleomycin), Raynaud's phenomenon, keratosis inflammation, Mithramycin Flush; An acneiform rash is found with the epidermal growth factor receptor inhibitors such as cetuximab and efitinib. These papules and pustules resemble acne although the typical comedone of acne is undetected in association with these drugs. Raynaud's phenomenon has been described in patients receiving bleomycin and vinblastine. Moreover, Mithramycin Flush has been described in patients treated with mithramycin.

Complications of immunosuppression: Skin infections and tumors. Administration of a chemotherapy agent in patients with autoimmune disorders such as scleroderma or lupus can develop the appearance of a circular red scaly rash. This is related to the drug but also involves the autoimmune process itself. Potential explanations for the increased incidence of malignancies in cancer patients, renal transplant patients, and other chronically immunosuppressed individuals embrace the direct cellular or oncogenic effect of the drugs being used, loss of $\mathrm{T}$ suppressor cell function, the existence of an oncogenic virus that is helped by immunosuppression, or the potentiation of environmental carcinogens by immunosuppression. In animal studies the alkylating agents and anthracycline antibiotics are directly oncogenic in healthy tissue. Other drugs, such as the antimetabolites and cyclophosphamide, are "co-oncogenic" and increase tumorigenic effects of other carcinogens.

\section{Palliative cosmetics}

The use of cosmetics to relieve some of the discomfort caused by the side effects of chemotherapy can be a strategy to maintain adherence to treatment [5].

The supply of palliative cosmetics can be fundamental in maintaining patient self-esteem by reducing some of the side effects, since hair, skin and nails are tissues with high rates of cell division, extremely affected by cancer treatments [39]. 
The selection of the active ingredients that best fit the needs of patients during cancer treatments must be made by analyzing the skin changes resulting from them. Because the skin is sensitized, development of products in a line for these patients should be focused on ingredients with moisturizing, soothing, antibacterial and healing properties. Hypoallergenic, alcohol-free and fragrance-free formulations should also be prioritized [39].

Active ingredients: a) Mild surfactants: Decyl Glucoside: Natural anionic surfactant with excellent dermal compatibility [39].

Coco-glucoside and Glyceryl Oleate: Its composition is similar to the lipid layer of the skin, preventing excessive dryness caused by cleansing agents [39].

\section{b) Humectants:}

- Butyrospermun Parkii (Shae) Butter: Antioxidant and antiinflammatory agent known for its ability to hydrate, smooth and promote skin and hair softness [39].

- Propanediol (and) Rhodosorus Marinus Extract: Biotechnological active that promotes the repair of the epidermal matrix and, therefore, is able to decrease neuroinflammatory effects. It acts in the inhibition of TRPV1 (a marker linked to longevity and hypoalgesia), IL-A and NFG [39].

- Propanediol: A very mild derivative of corn glucose with low potential to cause irritation. It can be used as a carrier for active ingredients, sensory enhancer, solvent, preservative enhancer and antimicrobial [39].

- Biosaccharide Gum-1: It has film-forming activity and improves the sensation of the skin. Stimulates longevity proteins, regulates inflammation markers and epidermal differentiation [39].

- Rosmarinus Officinalis (Rosemary) Leaf Extract: Can be used to stimulate hair growth and has anti-inflammatory, antiseptic, astringent, healing, and refreshing properties [40].

- D-Panthenol: Possesses healing effects related to the structure and function of living tissues and has a moisturizing action [41].

c) Soothing: Biosaccharide Gum-2: It has anti-inflammatory properties and protects the skin from external aggressors that accelerate aging. In addition to stimulating the production of $\beta$-endorphins, molecules that promote well-being [39,42].

- Safflower Oil/Palm Oil Aminopropanediol Esters: It has antiinflammatory and restructuring activity due to its ceramidelike structure, capable of protecting skin with damaged integrity $[39,43]$.

- Aloe Barbadensis Leaf Extract: Has anti-inflammatory, healing, regenerative, moisturizing and emollient properties [44].

- Chamomilla Recutita (Matricaria) Flower Oil: It has antiinflammatory, antiseptic, calming and refreshing properties. It also has a pleasant aroma [45].

d) Prebiotics: Alpha-Glucan Oligosaccharide: Selective stimulant of beneficial flora to the detriment of pathogenic and undesirable. Has protective and repairing action on the skin $[39,46]$.

- Alpha-glucan oligosaccharide (and) Polymnia sonchifolia root juice (and) Maltodextrin (and) Lactobacillus: Contains pre and probiotics that improve the appearance and comfort of sensitive and dry skin, promotes the growth of beneficial bacteria and reinforces the skin's natural defenses $[39,47]$.

- $\quad$ Propanediol (and) Aqua (and) Rhamnose (and) Glucose (and) Glucuronic Acid: Its chemical structure similar to that of Teflon ${ }^{\mathrm{Tx}}$ inhibits the adhesion of undesirable and pathogenic bacteria, maintaining the balance of flora and, therefore, neutralizing odors. It also has anti-inflammatory and soothing action $[39,48,49]$.

Formulations: In addition to the incorporation of the active ingredients mentioned above, formulations with the most acidified $\mathrm{pH}$ are a good option because they increase the hydration of the stratum corneum and reduce loss of transepidermal water. Cosmetics with $\mathrm{pH}$ ranging from 5.0 to 5.5 will maintain an appropriate level of hydration in the stratum corneum, which can help reduce symptoms related to skin dehydration.

Through clinical studies by the Instituto Dermatológico de San Gallicano, volunteers underwent 3 weeks of tests with cosmetic products with $\mathrm{pH}$ 5.5. The obtained results were reduction of transepidermal water loss, increased hydration of the stratum corneum, increased surface lipids, and improved epidermal basal barrier function [12].

The proper performance of the barrier function prevents skin infections and the worsening of adverse skin reactions caused by chemotherapy. Lack of water in the stratum corneum, unregulated epidermal hyperproliferation, and inadequate lipid synthesis are factors that trigger dehydrated skin with this impaired function [12].

Cleansing is an essential step to remove particles, oils, microorganisms and substances with foul smell. However, the removal of this dirt by excessive use of soaps causes dehydration of the horny extract [12]. Thus, it is interesting to use a milder cleansing foam formulation followed by a moisturizing cream with $\mathrm{pH}$ ranging between 5.0 to 5.5. Tables 1 and 2 contain the suggested formulations for soft foam and a moisturizer, respectively.

It is noteworthy that the two formulations do not contain ethyl alcohol, fragrance or more aggressive preservatives and surfactants. Therefore, they will be smoother for patients undergoing chemotherapy. Lactic acid has been added to make them more acidic and, thus, will make improvements in the horny extract as mentioned above in this topic.

Table 1: Suggested formulation for a mild foam for patients undergoing cancer treatment.

\begin{tabular}{|l|c|c|}
\hline \multicolumn{3}{|c|}{ Soft Cleansing Foam for cancer treatment patients* } \\
\hline Component & Function & $\%$ \\
\hline Aqua & Vehicle & qsp \\
\hline EDTA Na & Sequestrant & 0,05 \\
\hline Pantenol & Humectant & 5,0 \\
\hline Glycerin & Humectant & 5,0 \\
\hline Xanthan gum & Thickener & 0,2 \\
\hline $\begin{array}{l}\text { Propanediol (and) } \\
\text { Rhodosorus Marinus Extract }\end{array}$ & Active and preservative & 2,0 \\
\hline Decyl glucoside & Surfactant & 3,0 \\
\hline Cocoamidopropil Betaine & Surfactant & 4,0 \\
\hline Aloe Barbadensis Leaf Extract & Soothing agent & 5,0 \\
\hline Lactic acid & Ph correcting (5.0-5.5) & qsp \\
\hline $\begin{array}{l}\text { Phenoxyethanol (and) } \\
\text { Caprylyl Glycol }\end{array}$ & Preservative & 1,0 \\
\hline
\end{tabular}

*Requires use of specific packaging. 
Table 2: Suggested formulation for a moisturizing lotion for patients undergoing cancer treatments.

\begin{tabular}{|l|c|c|}
\hline \multicolumn{2}{|c|}{ Moisturizer for cancer treatment patients } \\
\hline Component & Function & $\%$ \\
\hline Aqua & Vehicle & Qsp \\
\hline EDTA Na ${ }^{2}$ & Sequestrant & 0,1 \\
\hline Pantenol & Humectant & 1,0 \\
\hline Aristoflex AVC & Thickening & 0,6 \\
\hline Glycerin & Humectant & 8,0 \\
\hline Xanthan gum & Thickening & 0,2 \\
\hline Euphorbia cerifera (Candelilla) wax & Emollient & 1,0 \\
\hline Ceteareth-20 & Emulsifier & 2,0 \\
\hline Cetearyl Alcohol & Emulsifier & 6,0 \\
\hline Shea Butter & Emollient & 5,0 \\
\hline Vitamin E & Antioxidant & 0,7 \\
\hline $\begin{array}{l}\text { Chamomilla recutita (Matricaria) } \\
\text { Flower Oil }\end{array}$ & Soothing agent & 1,0 \\
\hline Safflower Oil/Palm & Soothing agente & 0,5 \\
\hline Oil Aminopropanediol Esters & & 1,0 \\
\hline Coco-caprylate (and) Caprate & Emollient & 0,8 \\
\hline $\begin{array}{l}\text { Alpha-glucan oligosaccharide } \\
\text { (and) Polymnia sonchifolia root } \\
\text { juice (and) Maltodextrin (and) } \\
\text { Lactobacillus }\end{array}$ & Preservative & 1,0 \\
\hline Lactic acid & & 1,0 correcting (5,0-5,5) \\
\hline $\begin{array}{l}\text { Phenoxyethanol (and) Caprylyl } \\
\text { Glycol }\end{array}$ & Hsp \\
\hline
\end{tabular}

\section{Conclusion}

Based on the literature consulted, it was observed that the use of suitable cosmetic products proved to be beneficial in the treatment of sensitized skin in patients undergoing chemotherapy. Products with adequate active ingredients, slightly acidified $\mathrm{pH}$, fragrance free, alcohol free, and with milder preservatives are better suited.

\section{References}

1. Alley E, Green R, Schuchter L (2002) Cutaneous toxicities of cancer therapy. Curr Opin Oncol 14: 212-216.

2. Branzan AL, Landthaler M, Szeimies RM (2005) Skin changes with chemotherapy. Hautarzt 56: 591-602.

3. Wagner LI, Lacouture ME (2007) Dermatologic toxicities associated with EGFR inhibitors: the clinical psychologist's perspective. Impact on health-related quality of life and implications for clinical management of psychological sequelae. Oncology (Williston Park) 21: $34-36$

4. Fabbrocini G, Cameli N, Romano MC, Mariano M, Panariello N, et al. (2012) Chemotherapy and skin reactions. J Exp Clin Cancer Res 31: 50 .

5. Fluhr JW, Miteva M, Primavera G, Ziemer M, Elsner P, et al. (2007) Functional Assessment of a Skin Care System in Patients on Chemotherapy. Skin Pharmacol Physiol 20: 253-259.

6. Guillot B, Bessis D, Dreure O (2004) Mucocutaneous side effects of antineoplastic chemotherapy. Expert Opin Drug Saf 3: 579-587.

7. Bronner AK, Hood AF (1983) Cutaneous complications of chemotherapeutic agents. J Am Acad Dermatol 9: 645-663.
8. DeSpain JD (1992) Dermatologic toxicity of chemotherapy. Semin Oncol 19: 501-507.

9. Koppel RA, Boh EE (2001) Cutaneous reactions to chemotherapeutic agents. Am J Med Sci 321: 327-335.

10. Diegelmann RF, Evans MC (2004) Wound healing: an overview of acute, fibrotic and delayed healing. Front Biosci 9: 283-289.

11. (2017) Cancer treatment statistics. Cancer Research UK.

12. DeHaven C (2014) Chemotherapy and Radiotherapy Effects on the Skin. Plast Surg Nurs 34: 192-195.

13. Viale PH (2006) Chemotherapy and Cutaneous Toxicities: Implications for Oncology Nurses. Semin Oncol Nurs 22: 144-151.

14. Hood AF (1986) Cutaneous Side Effects of Cancer Chemotherapy. Med Clin North Am 70: 187-209.

15. Souza VM de, Junior DA (2016) Ativos dermatológicos: dermocosméticose nutracêuticos. ( $1^{\text {st }}$ edtn) São Paulo, Brazil, 32-40.

16. Gupta MA, Gilchrest BA (2005) Psychosocial aspects of aging skin. Dermatol clin 23: 643-648.

17. Fitzpatrick T, Freedberg I (2003) Dermatology in general medicine. ( $6^{\text {th }}$ edtn) Whitehouse Station: McGraw-Hill, Health Professions Division, USA.

18. Urmacher C (1995) Normal Skin in Sternberg SS. Histology for Pathologists, New York Raven Press, 381-398.

19. Leonardi GR (2004) Cosmetologia aplicada. Editora Medfarma, São Paulo.

20. Contran RS, Kumar V, Robbins CT (2001) Patologia estrutural e funcional. Guanabara Koogan, Rio de Janeiro, 44-100.

21. Davies MJ (1990) A macro and micro view of coronary vascular insult in ischemic heart disease. Circulation 82: II38-1146.

22. Santoro MM, Galdino G (2005) Cellular and molecular facets of keratinocyte reepithelization during wound healing. Exp Cell Res 304: 274-286.

23. (2019) Wound Healing 201-The Master Class. Pilonidal Support Alliance.

24. Kalia S, Avérous L (2011) Biopolymers: Biomedical and Environmental Applications. Wiley, Salem, USA, 614.

25. Lefkovits J, Plow EF, Topol EJ (1995) Platelet glycoprotein IIb/IIla receptors in cardiovascular medicine. N Engl J Med 332: 1553-1559.

26. O que ê câncer? INCA Instituto Nacional de Câncer.

27. What is Cancer?-SEER Training.

28. Cancer Terms-SEER Training.

29. Cancer Development-CancerQuest.

30. Cooper GM (2000) The Cell: A Molecular Approach. $2^{\text {nd }}$ edtn Sunderland (MA), Sinauer Associates, USA.

31. (2019) DNA Mutation and Repair.

32. (2019) Mechanisms of DNA Repair-NEWS.

33. Clancy S (2008) DNA damage \& repair: mechanisms for maintaining DNA integrity. Nature Education 1: 103.

34. (2020) Signs and Symptoms of Cancer. American Cancer Society.

35. Como surge o câncer- INCA.

36. Arruebo M, Vilaboa N, Sáez-Gutierrez B, Lambea J, Tres A, et al. (2011) Assessment of the evolution of cancer treatment therapies. 
Cancers (Basel) 3: 3279-3330.

37. (2020) Cancer Treatment- Mayo Clinic.

38. Souza VM de, Junior DA (2016) Ativos dermatológicos: dermocosméticos e nutracêuticos. ( $1^{\text {st }}$ edtn) São Paulo, Brazil, 67.

39. (2018) Cosméticos para pacientes oncológicos- Cosmetics Online.

40. Rosemary Eco- Prospector ${ }^{\circ}$.

41. (2015) D-Panthenol USP-Prospector

42. Rhamnosoft ${ }^{\circledR}$ HP- Prospector ${ }^{\circledR}$.

43. ' $\Omega 6$ Ceramide $^{\circledR}$-Prospector'.
44. Santos LPS, Santos MCS, Morbeck NBM (2019) Estética Humanizada nos cuidados paliativos em pacientes oncológicos: da massoterapia ao cosmético uma revisão. In: Anais da IX Jornada de Estética e Cosmética do CEULP/ULBRA 4: 6-19.

45. Chamomile Eco- Prospector

46. BioEcolia ${ }^{\circledR}$ - Prospector ${ }^{\circ}$.

47. Ecoskin ${ }^{\circledR}$ - Solabia Cosmetics Group.

48. Teflose $^{\circledR}$ - Prospector ${ }^{\circledR}$.

49. Teflose ${ }^{\circledR}$ - Solabia Cosmetics Group. 